\title{
Israeli researchers eye simple test for multiple sclerosis
}

Researchers are set to begin clinical trials of a blood test that can easily distinguish between severe multiple sclerosis (MS) and a benign form of the disease.

Up to 25 percent of those diagnosed with MS have a benign form of the disease and may be in remission for ten years or more. But because there is no way to identify benign MS in the early stages, those patients needlessly take medications and experience anxiety for years. Conversely, identifying those with severe MS means that they could receive aggressive treatment at the outset.

"What is needed is an inexpensive, easily obtained barometer for monitoring disease activity in these patients," says Stuart Cook, professor of neuroscience at the University of Medicine and Dentistry of New Jersey and the trial's lead investigator. Most methods are either too expensive or not sensitive enough, he says.

The test, developed by Israeli company
Glycominds, is based on glycans - the sugars found inside and on the surface of human cells-which play a crucial role in autoimmune and inflammatory diseases. Using the company's library of glycans, the researchers identified an antibody to a certain glycan that is elevated in the cells of people with MS and determined its level in more than 200 patients. "We proved that we have a specific marker [for MS severity] based on the level of [specific] antibodies on these specific sugars," says Glycominds chief executive Avinoam Dukler.

The claim will be tested in 385 people at 80 centers across the US, Canada and Israel and is the largest trial of patients with 'clinically isolated syndrome'-in which they exhibit the first signs of MS and magnetic resonance imaging indicates they have a high possibility of MS - who have not begun treatment.

Participants will not receive any treatment for six months. "We will predict from taking their blood at the first presentation whether there will be a second presentation within six months," says Dukler. Participants will then be monitored for a further eighteen months, with blood taken every six months.

An accurate test could be invaluable to MS patients, but researchers must proceed carefully, says Jack Burks, chief medical officer of the Multiple Sclerosis Association of America. If the test wrongly diagnoses patients as having only benign disease, for instance, they would miss the necessary treatment, he notes. "The brain cells they lose by foregoing treatment are lost forever."

If the blood test proves accurate at the first six-month point, a product could be on the market as early as 2006. Glycominds is also working on a glycan-based test for distinguishing between Crohn's disease, ulcerative colitis and irritable bowel syndrome.

Tania Hershman, Jerusalem

\section{Reports of SIDS-virus link greatly exaggerated, experts say}

International newspapers in September made much of a report showing that a recently discovered virus was present in tissues from a pair of so-called 'crib deaths.' But even the researchers who discovered the virus remain skeptical that it might have caused the deaths.

Doctors initially attributed the deaths of two infant girls in Wisconsin in 2003 to Sudden Infant Death Syndrome (SIDS). But when tissue samples from both infants tested positive for the previously unknown human parechovirus 3 (HPEV-3), the media began speculating about a link between the virus and SIDS.

But Gerald Sedmak, the Milwaukee virologist who first detected the new agent, is unconvinced. "We never made a big deal out of a possible link to SIDS," says Sedmak. "What we thought was most interesting was that these were the first cases of HPEV-3 in the United States."

HPEV-1, HPEV-2 and the newly discovered HPEV-3 are members of the picornavirus family of single-stranded RNA agents. Parechoviruses have been implicated in flaccid paralysis, encephalitis, gastroenteritis and respiratory infections. Researchers in Aichi, Japan, first reported HPEV-3 in a study published in January (J. Gen. Virol. 85, $391-398 ; 2004)$. They isolated the virus from samples taken from a 1-year-old girl with diarrhea, fever and transient paralysis.

But despite HPEV-3's pathogenic profile, there have been no reports of deaths, infant or adult, caused by the virus in Japan, notes lead researcher Miyabi Ito. When the scientists tested children in Aichi for antibodies to HPEV-3, they found that by the time children enter elementary school, more than $80 \%$ of them test positive for the virus. "With such a high prevalence and no reported deaths, we considered [the virus] to be very infectious, but relatively benign," says Ito.

The Milwaukee health department found the virus during a routine screening to track bacterial and viral involvement in unexplained deaths. But viral infectionmost commonly by enteroviruses and adenoviruses, cytomegalovirus and influenza-is not uncommon in SIDS deaths, Sedmak notes. "We see about a $30 \%$ viral infection rate in SIDS," he says.

HPEV-3 was not previously on health officials' radar, and questions remain about the route of transmission and the extent of the Milwaukee outbreak. A third case-tissue taken from a 16-month-old whose death was not classified as SIDS - tested positive for HPEV-3 in Milwaukee in August, and confirmation on a fourth is pending. Since 1987 , the surveillance program that detected the HPEV-3 infections has also reported 17 infant deaths, including a number of nonSIDS cases, in which HPEV-1 was found in tissue samples, suggesting that the viruses might be relatively common.

Meanwhile, researchers are examining factors ranging from genetic mutations to

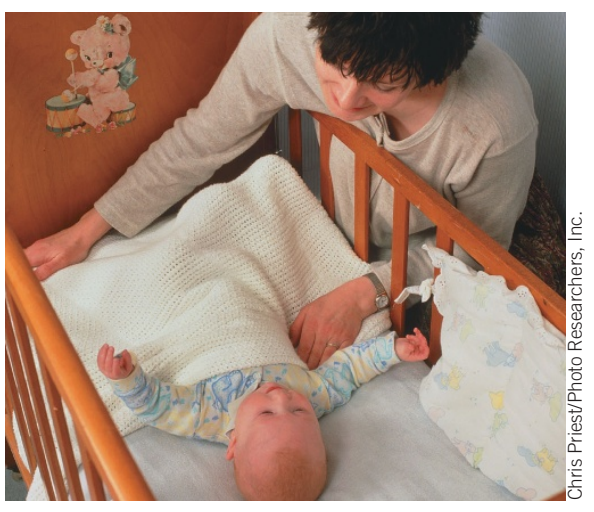

Crib deaths, which claim thousands of lives each year, remain a mystery.

uterine proteins and the mother's economic status to determine SIDS risk, but have not yet found a single conclusive cause. In the 1990s, SIDS was famously linked to a prone sleeping position, and subsequent national 'Back to Sleep' programs are credited with significantly reducing SIDS deaths. But many infant deaths remain unexplained.

"Some people may feel there's ultimately a single explanatory factor behind SIDS deaths, but most understand it as a catch-all diagnosis for deaths caused by a whole range of undetermined factors," says Kurt Nolte, a forensic pathologist at the University of New Mexico. "It's one of those things where you just have to keep chipping away."

Douglas Sipp, Kobe 